\title{
The portable glostavent: Speed of assembly and successful ventilation for naive operators
}

\author{
Jacob Benrud, Shannon Grap, Allison Weinstock and Julie Vuong* \\ Department of Anesthesiology, Penn State Hershey Medical Center, Hershey, PA, USA
}

\begin{abstract}
The increasing interest in,and opportunities for, global anesthesia outreach have revealed a need for familiarity with medications, anesthesia techniques, and anesthesia delivery systems often never before encountered by first world anesthesiologists. A medical mission trip may be the first time an anesthesiologist handles a specific type of anesthesia delivery system, which is often rudimentary and may be in a state of disrepair. These life sustaining devices may be apt to malfunction; this propensity is especially dependent on the resources inherent to the local environment and can lead to potentially disastrous and unsafe events. The draw-over anesthesia technique is ideally suited for such environments because of its simple and safe design. The objective of our study was to determine whether anesthesiologists without previous experience using a portable version of the Glostavent (GV, Diamedica Ltd, Barnstaple, Devon, United Kingdom), could use the manufacturer's instructions to assemble the device and successfully ventilate a mannequin in a simulation scenario. Our study demonstrates that a novel draw-over anesthesia machine could use the manufacturer's instructions to assemble the device and successfully ventilate a mannequin in a simulation scenario. Our hypothesis was that naive operators, after a 10 minute self-training period, would be able to accomplish the task. Twenty nine of thirty participants (resident and attending anesthesiologists) were able to assemble the machine and successfully ventilate the mannequin within the ten minute test period. The results of our study demonstrate that this novel draw-over anesthesia machine can be easily assembled and its use learned by anesthesiologists who have never seen such a device before. These results suggest that this simple suitcase anesthesia machine is a worthy investment, especially for anesthesiologists who plan to participate in outreach anesthesia.
\end{abstract}

\section{Introduction}

Much of the world lacks resources to allow use of modern complex anesthesia machines in clinical practice. Aportable version of the Glostavent (GV, Diamedica Ltd, Barnstaple, Devon, United Kingdom), the Diamedica Portable Anesthesia Machine,(DPA 03 ${ }^{\mathrm{TM}}$ ) (Diamedica Ltd, Barnstaple, Devon, United Kingdom, Figures 1 and 2) offers a self-contained solution to provide anesthesia in disadvantaged environments, including disaster settings. The DPA $03^{\mathrm{TM}}$ does not require the use of electricity or compressed medical gases, including oxygen, in order to deliver inhaled anesthesia and provide ventilatory assistance [1]. Draw-over anesthesia machines such as the DPA $03^{\mathrm{TM}}$ have been utilized by military personnel and anesthetists in developing countries for years, however, interest in this portable anesthesia machine has increased for use in medical outreach due to its simplicity and safety [1-3].

This research focused not on the merits of the DPA $03^{\mathrm{TM}}$, but rather onits assembly and use by anesthesiologists without previous experience using this machine. Our hypothesis was that naive operators would be able to use basic instructions provided by the manufacturer with the machine to assemble the DPA $03^{\mathrm{TM}}$ and then successfully ventilate a mannequin, all within a reasonably short period of time. If our hypothesis can be substantiated, by demonstrating success of quick assembly and use of the DPA $03^{\mathrm{TM}}$ by naive operators, then we will have further demonstrated its utility for use in medical mission outreach and austere environments.

\section{Objective}

The primary endpoints were time to successful assembly of the DPA $03^{\mathrm{TM}}$ during a 10 minute self-training period, and time to confirmed ventilation of a mannequin, in a simulated scenario where the participant began with the DPA $03^{\mathrm{TM}}$ unassembled in its suitcase.

Secondary endpoints were satisfaction of the participants with the provided instructional materials, the confidence of the participants in their ability to use the DPA $03^{\mathrm{TM}}$ in the future, and participant's suggestions for helping naïve operators successfully get the device from suitcase to successful use.

\section{Methods}

This was a prospective, observational study. Anesthesiology residents and faculty currently in practice at the Penn State HersheyMedical Center Department of Anesthesiology were asked to participate. Each participant was screened to ensure they were naïve to the DPA $03^{\mathrm{TM}}$. Each participant had 30 minutes available to participate in the research. The research team member served as the evaluator during the activity.

\section{Self-training period}

Each participant was given an introduction stating: "You have just arrived at a remote destination for outreach anesthesia and one of the pieces of equipment that youwill need to use is theportable DPA $03^{\mathrm{TM}}$ ".

Correspondence to: Julie Vuong, Department of Anesthesiology, H187, Penn State Hershey Medical Center, Hershey, PA 17033, USA, Tel: (608) 780-9871; E-mail: jbenrud@hmc.psu.edu

Key words: anesthesia, draw-over anesthesia, glostavent, limited resources, outreach

Received: April 05 2015; Accepted: April 29, 2015; Published: May 01, 2015 


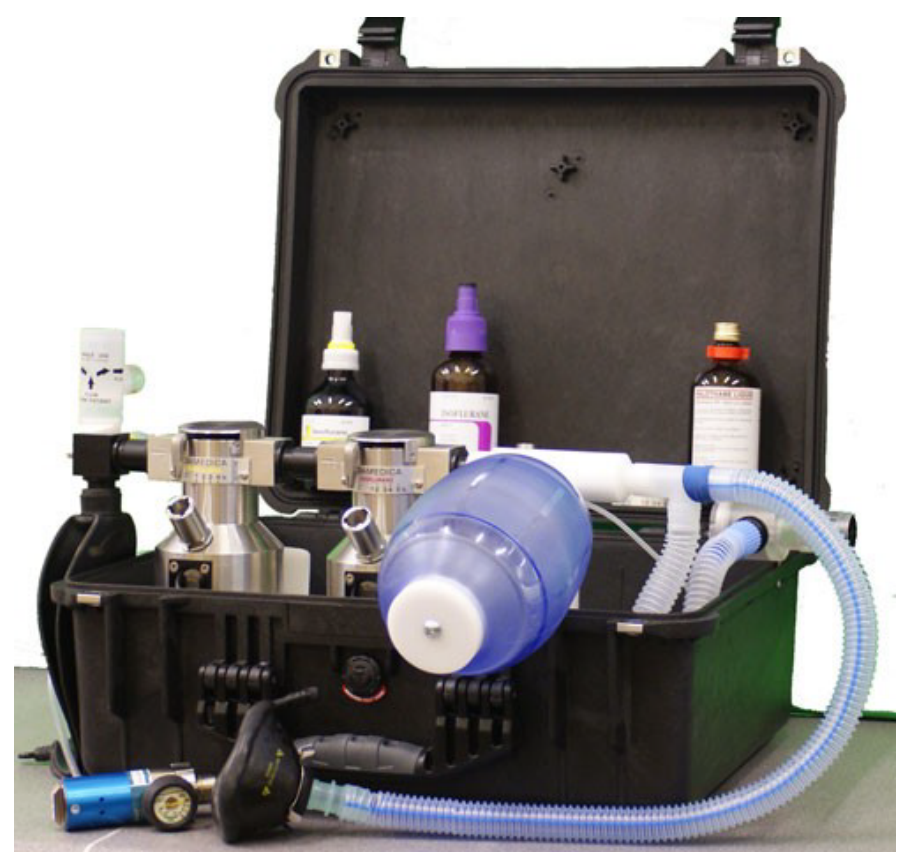

Figure 1. DPA 03TM (Copyright Diamedica).

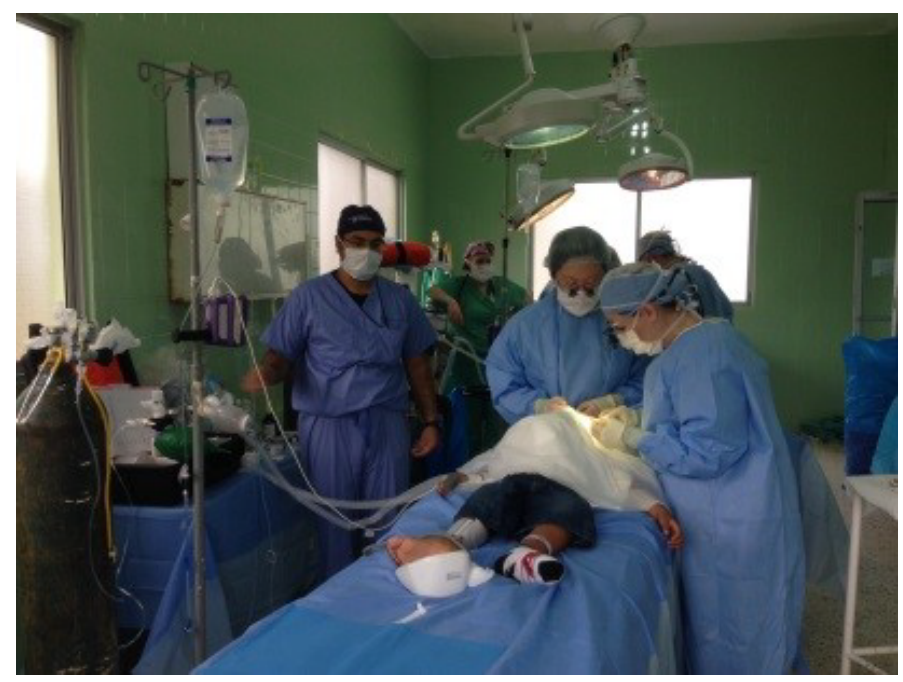

Figure 2. DPA 03TM in use in an austere environment.

The unassembled DPA $03^{\mathrm{TM}}$ was given to the participant in its carrying suitcase, as provided by the manufacturer, and they had 10 minutes for familiarization with the manufacturer's instructions and practicing device assembly in preparation for its use.

The self-training time period began when the participant opened the suitcase containing the DPA $03^{\mathrm{TM}}$. Timing was stopped when the participant successfully assembled the machine, or the10 minute time frame had passed. The evaluator determined correct DPA $03^{\mathrm{TM}}$ assembly. The evaluator disassembled the device and returned it to the suitcase before continuing to the next step, the simulation session.

\section{Simulation session}

To determine the time to assemble the DPA $03^{\mathrm{TM}}$ and confirm ventilation of a mannequin using the properly constructed machine, the participant was asked again to assemble the DPA $03^{\mathrm{TM}}$ following the self-training period. Although the DPA $03^{\mathrm{TM}}$ comes with a mask for ventilation, we chose to use an endotracheal tube for purposes of proving successful use of the DPA $03^{\mathrm{TM}}$ rather than proficiency with mask ventilation on the fairly rigid mannequin head. The endotracheal tube was placed in the mannequin by a member of the research team, prior to the arrival of the subject, and proper position and function was ensured prior to the start of the research activity. The evaluator began timing when the participant opened the DPA $03^{\mathrm{TM}}$ suitcase. Timing was stopped when ventilation of the mannequin was confirmed by evidence of lung inflation, or after 10 minutes had passed.

Following completion of the timed self-training and simulation sessions, the evaluator asked the participant questions regarding his or her impressions of the DPA $03^{\mathrm{TM}}$ and its use in the simulation scenario (Figure 3). The evaluator then shared the results with the participant.

\section{Results}

Thirty subjects participated in the study, but one was excluded because of failure to assemble and ventilate the mannequin in both the self-teaching and simulation session time periods. Six of the 29 participants $(21 \%)$ were unable to put the machine together during the initial self-training period, but all $29(100 \%)$ successfully assembled the device and ventilated the mannequin during the simulation session. Thus, all but one was able to learn from the self-training handson period, and succeed with proper assembly and ventilation of the mannequin, using the provided manufacturer's instructions. The average time for all participants for initial learning was 369 seconds (6 minutes, 9 seconds) and the average time for successful assembly during the simulation scenario was 109 seconds ( 1 minute, 49 seconds).

Record ID

Glostavent Self-instruction course participant evaluation

Date:

1. Level of Training:

$\bigcirc$ Resident

Faculty

2. How many years of anesthesia the participant has practiced, including training?

3. Time to assembly of the portable GV during the self-teaching period ( $\max 10 \mathrm{~min}$ ).

4. Was the portable GV assembled correctly during the self-teaching period? $\bigcirc$ Yes

$\bigcirc$ No

5. Time to confirmed ventilation of the mannequin during the simulation scenario (max 10 $\min )$

6. Would you feel comfortable assembling and using the portable GV, as packaged, in an urgent situation where the GV was your best resource for administering anesthesia? Y Yes

$\bigcirc$ No

7. Do you feel the self-teaching materials provided by the manufacturer of the GV in the suitcase are sufficient and effective for use of the GV in urgent situations in the field? $\bigcirc$ Yes

$\bigcirc$ No

8. What parts of the instructional material did you find to be the most useful?

$\bigcirc$ Images

9. If you were to use this machine in a high stress environment, are there any changes you would make to the machine setup, capabilities, or instructional material? ○Yes

$\bigcirc$ No

If answered yes for question 9, please describe any changes you would make.

Figure 3. Participant Survey. 
Stratification of participants based on experience in anesthesiology, the corresponding times needed to assemble the device during the self-training period, and the average times required for successful ventilation of the mannequin during the simulation session, are shown in Table 1. Residents in their final year of residency achieved the fastest average time to assembly during the self-training period and were, on average, 38 and 24 seconds faster than faculty members with 5-10 y or $>10$ y experience, respectively. Of the six participants who did not correctly assemble the machine during the self-training period, 4 were residents (1 CB, 1 CA-1, and 2CA-2's) while 2 were faculty with $>10$ y experience.

In the simulation session, the average time to ventilation did not differ between all residents and all faculty. However, final year residents achieved the fastest average time, which was 22 seconds faster than faculty with 5-10 y experience, who were the second fastest group (Figure 4 ). The only other group to achieve an average time to ventilation that was $<100$ seconds was the CA-2 residents. CA-1 residents had the longest average time and were 33 seconds slower than the second slowest group, faculty with $>10$ y experience.

Of the twenty-nine participants, 27 (93\%) indicated that they would feel comfortable assembling and using the DPA $03^{\mathrm{TM}}$, as packaged, in an urgent situation where the DPA $03^{\mathrm{TM}}$ was available for administering anesthesia. Twenty-one of the participants (72\%) indicated that they thought that the self-teaching materials provided by the manufacturer of the DPA $03^{\mathrm{TM}}$ in the suitcase are sufficient

Table 1. Average time to Assembly and Ventilation Among Participant Types.

\begin{tabular}{|l|c|c|}
\hline Participant type & $\begin{array}{c}\text { Average time to assembly } \\
\text { (self-training period, seconds) }\end{array}$ & $\begin{array}{c}\text { Average time to Ventilation } \\
\text { (simulation session, seconds) }\end{array}$ \\
\hline CB & 335 & 109 \\
\hline CA-1 & 413 & 168 \\
\hline CA-2 & 465 & 97 \\
\hline CA-3 & 312 & 53 \\
\hline Faculty 5-10 yr & 350 & 75 \\
\hline Faculty 10+yr & 336 & 135 \\
\hline All Residents & 384 & 109 \\
\hline All Faculty & 343 & 108 \\
\hline All Participants & 369 & 109 \\
\hline
\end{tabular}

$\mathrm{CB}(\mathrm{n}=6), \mathrm{CA}-1(\mathrm{n}=5), \mathrm{CA}-2(\mathrm{n}=4), \mathrm{CA}-3(\mathrm{n}=3)$, Faculty with 5 to 10 years of experience $(\mathrm{n}=5)$, Faculty with greater than 10 years of experience $(\mathrm{n}=6)$, all residents $(\mathrm{n}=18)$, all faculty $(\mathrm{n}=11)$, all participants ( $\mathrm{n}=29$ as one participant was excluded).

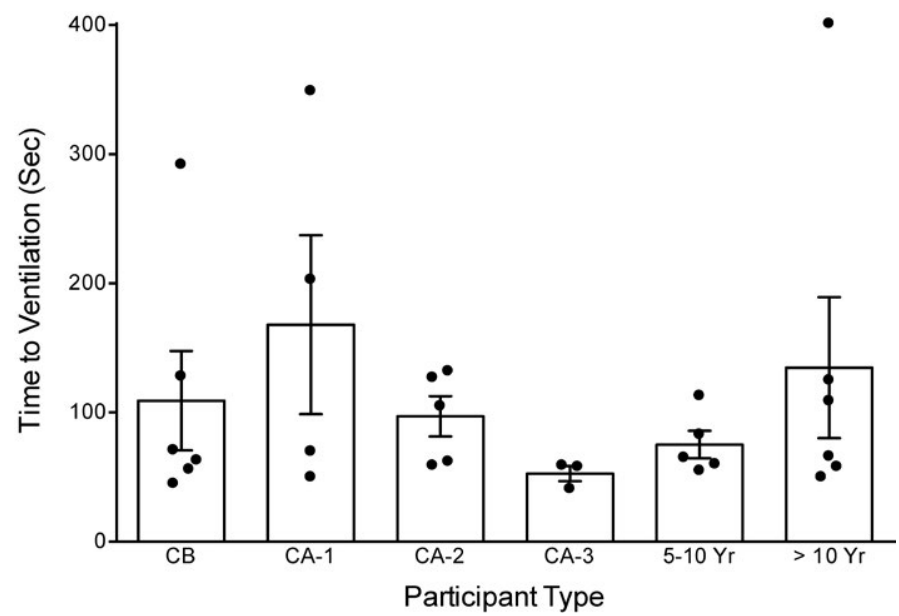

Figure 4. Time to successful ventilation among participant types.

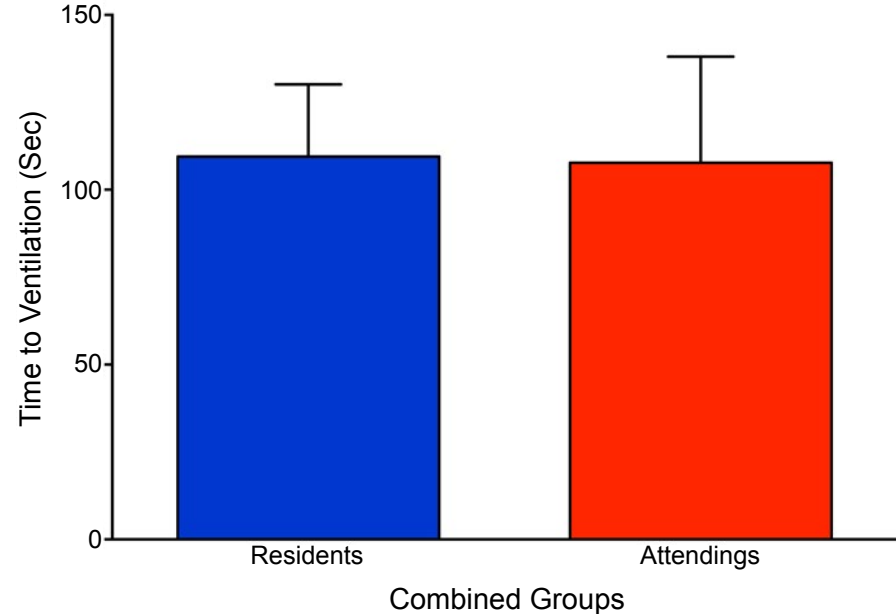

Figure 5. Time to successful ventilation among residents and attendings.

and effective for use of the DPA $03^{\mathrm{TM}}$ in urgent situations in the field. Twenty-sixparticipants (90\%) indicated that they felt that the images included with the instructions were the most useful when compared to the written instructions. Nineteen participants (66\%) indicated that they would make changes to the instructions, set up, or assembly. When those nineteen participants were asked what changes they would make, 9 (47\%) made statements saying that they would want better labeling of parts for assembly; of those 9, 7 participants (78\%) specifically mentioned the use of color coded parts. Ten participants (53\%) suggested in some form that the instructions should be improved upon and of those 10, 7 participants (70\%) stated that they would like to see better labeling of the instructions. Other suggestions included using simpler packaging, changing connector sizes to prevent incorrect assembly, having instructions in a frequently asked questions or trouble shooting format, step-by-step diagrams included in the instructions, and having the vaporizer more secure (Figure 5).

\section{Discussion}

To our knowledge, this is the first study evaluating the assembly and successful ventilation, bynaive operators, using the DPA $03^{\mathrm{TM}}$ in a simulation scenario. The DPA $03^{\mathrm{TM}}$ has been previously used with favorable results, in Hoima Referral Hospital in Uganda, for caesarian sections by Read and Taylor in 2012 [1]; however, no one has yet studied whether anesthesiologists new to it could quickly assemble and use the device, in simulation, with only self-training. We found encouraging results regarding the ability of practicing anesthesiologists to assemble this new anesthesia machine. It appears that even if an anesthesiologist in the initial encounter fails to assemble the DPA $03^{\mathrm{TM}}$, nearly everyone can successfully assemble and prepare it for operative use, in a timely manner, during a second encounter. Furthermore, an overwhelming majority (93\%) of participants stated that they would feel comfortable assembling and using the DPA $03^{\mathrm{TM}}$, as packaged, in an urgent situation where the DPA $03^{\mathrm{TM}}$ was available for administering anesthesia. This supports our hypothesis that the DPA $03^{\mathrm{TM}}$ is a good solution for use in outreach anesthesia for those unfamiliar with the device who wish to avoid use of local equipment in questionable repair.

Over two-thirds of participants (72\%) agreed that the self-teaching materials provided by the manufacturer of the DPA $03^{\mathrm{TM}}$ in the suitcase are sufficient and effective for use of the DPA $03^{\mathrm{TM}}$ in urgent situations in the field; however, $34 \%$ still contended that there could be improvements made to the instructions, and most of these participants 
(7 of 10) stated that the labeling on the instructions themselves could be improved. Thus, the instructions may be sufficient, but they are not yet perfect.

Our study was limited, to the small sample size available at the Penn State Hershey Medical Center, thus potentially limiting its external validity. Our sample size was; however, justified given similar self-learning studies [4]. Although the sample size was relatively small, most participants were able to successfully assemble and ventilate the mannequin given only a short period of time. Another limitation of this study is that the participants only took part in a simulation. The actual use and confidence in use of this anesthesia machine may be more limited when faced with its use in a real encounter.

\section{Conclusion}

We found encouraging results regarding the ability of practicing anesthesiologists to assemble a new anesthesia machine built for austere and disadvantaged environments. It appears that even if anesthesiologists do not successfully assemble the DPA $03^{\mathrm{TM}}$ during an initial brief encounter, nearly all can successfully assemble and utilize this precious life-saving machine for operative use during a second encounter. Often, in the outreach anesthesia setting, pipeline oxygen required for modern machines may not exist, skilled technicians for proper maintenance may not be available, and even electricity and oxygen tanks may be in fluctuating supply. In these arenas, anesthetists may very well be better served by an economical (in cost of the machine) and safe machine, the use of which can be quickly learned, and which can operate despite a lack of supplemental oxygen and electricity than by a foreign, leaky machine dependent on pressurized oxygen.

\section{References}

1. Read E, Taylor E (2012) Portable Diamedica Glostavent: an anaesthetic machine for the itinerant anaesthetist. Br J Anaesth 109: 648-649. [Crossref]

2. Pearson A, Eltringham R (2011) 'How to' article number 1: draw-over anaesthesia World Anaesthesia News. 12: 5-9.

3. Tully R, Eltringham R, Walker IA, Bartlett AJ (2010) The portable Glostavent: a new anaesthetic machine for use in difficult situations. Anaesth Intensive Care 38: 10851089. [Crossref]

4. Pott LM, Santrock D (2007) Teaching without a teacher: developing competence with a Bullard laryngoscope using only a structured self-learning course and practicing on a mannequin. J Clin Anesth 19: 583-586. [Crossref]

Copyright: (C2015 Benrud J. This is an open-access article distributed under the terms of the Creative Commons Attribution License, which permits unrestricted use, distribution, and reproduction in any medium, provided the original author and source are credited. 